\title{
STRONG CONVERGENCE OF NUMERICAL SOLUTIONS TO DEGENERATE VARIATIONAL PROBLEMS
}

\author{
R. A. NICOLAIDES AND NOEL J. WALKINGTON
}

\begin{abstract}
Numerical approximations of strongly degenerate variational problems of the form $J(u)=\int_{0}^{1} F\left(u^{\prime}\right)+(u-f)^{2}$ are considered, where $F$ is assumed convex but may have intervals where $F^{\prime \prime}=0$. It is shown that, in spite of the degeneracy, natural numerical approximations still converge in $W^{1, p}$. Rates in weaker norms and the connection with nonconvex variational problems are also considered.
\end{abstract}

\section{INTRODUCTION}

We consider a one-dimensional variational problem of the form

$$
J(u)=\int_{0}^{1} F\left(u^{\prime}\right)+(u-f)^{2},
$$

where $F$ is convex but vanishes on a finite interval. In this situation, minimizing sequences need not converge strongly in $W^{1, p}(0,1)$. For example, if $f=0$ and $F$ is the convexification of the double-well potential shown in Figure 1a, then the oscillatory sequence shown in Figure $1 \mathrm{~b}$ is minimizing. Clearly, the limit is zero; however, the derivatives only converge weakly to zero. While such undesirable behavior is possible in general, we show that the minimizing sequence, $\left\{u_{h}\right\}_{h>0}$, obtained by minimizing over piecewise linear functions on a uniform mesh having $N=1 / h$ nodes does converge strongly, and we obtain a rate in a weighted norm. The only other results we know of concerning problems with such degeneracies are given in French [5]. The results in [5] give strong convergence of the derivatives away from the degenerate set in a weighted norm (cf. $\S 3$ below), and, provided $F^{\prime}$ is nonzero on the degenerate set, it is shown that $\operatorname{sgn}\left(u_{h}^{\prime}\right)$ converges to $\operatorname{sgn}\left(u^{\prime}\right)$ on the degenerate set.

The problem considered here is motivated from problems in materials science where the bulk energies are not convex [1, 6, 7]. In this situation, it is known that any minimizing sequence will oscillate, so that strong convergence is never achieved. However, such sequences have associated Young measures

Received by the editor September 5, 1992 and, in revised form, December 2, 1993.

1991 Mathematics Subject Classification. Primary 65N15, 65N30.

Key words and phrases. Approximation of degenerate variational problems.

This work was supported by the Army Research Office and National Science Foundation through the Center for Nonlinear Analysis; the first author was supported by the United States Air Force under Grant AFSOR F49620-92-J-0133, and the second author by the National Science Foundation under Grant No. DMS-9002768. 


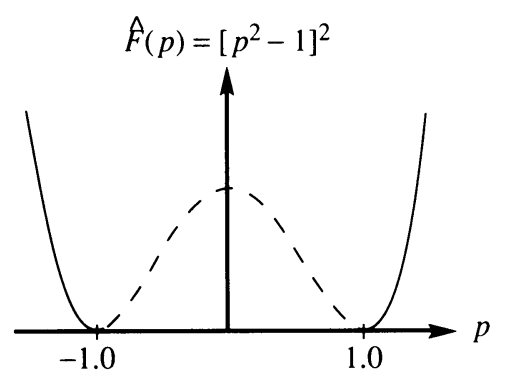

Figure 1a. Double well energy

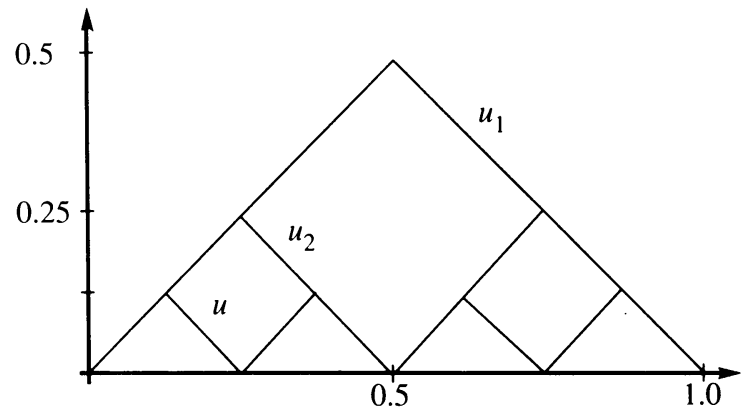

FIGURE 1b. A minimizing sequence

and weak limits, and recently the authors have proposed algorithms to compute these quantities without having to represent highly oscillatory functions on discrete meshes. In one dimension the convergence of these algorithms reduces to the analysis of the degenerate problem stated above. In $\$ 4$ we briefly review our algorithm and discuss the implications of the results proved below. Note that it is necessary to include the low-order term in the functional $J$ in order to get uniqueness. Moreover, without this term, minimizing sequences of the corresponding nonconvex problem would not necessarily develop fine-scale oscillations. This contrasts with higher-dimensional problems, where boundary conditions may "compete" with the energy resulting in oscillatory minimizing sequences [1].

The techniques used in our analysis are applicable to quite arbitrary functions $F$. However, to reduce the technical detail, we consider only the (convexification of the) inhomogeneous Young problem, where $F$ is the function shown in Figure 1a. The key step required to establish strong convergence is a "regularity" result, Theorem 2.1 , which shows that the degenerate set is essentially an interval. In this situation, a detailed analysis of the numerical solutions on the degenerate set is possible.

In the next section we introduce the discrete problem and show that the discrete solutions do converge strongly in the natural class of functions. In $\S 3$ we use Ekeland's lemma to establish a rate of convergence of the derivatives in a weighted norm, and finally in $\S 4$ the connection with algorithms to calculate Young measures is brought out. We conclude this section with some notation. $W^{m, p}(0,1)$ denotes the Sobolov space of functions whose $m$ th derivative is $p$ integrable, and $W_{0}^{m, p}(0,1)$ will denote the subspace whose traces vanish. Discrete solutions will be constructed on uniform meshes with $N=1 / h$ nodes, $h>0$, and the piecewise linear solutions constructed on such a mesh will be denoted by $u_{h}$, the continuous solution being denoted by $u$. By $C$ and $c$ we denote generic constants independent of $h$.

\section{StRong CONVERGENCE OF THE NUMERICAL SCHEME}

2.1. Continuous problem. The variational problem we consider is

$$
J(u)=\int_{0}^{1} F\left(u^{\prime}\right)+(u-f)^{2}, \quad u \in U=W_{0}^{1,4}(0,1),
$$

where $F$ is the convexification of $\widehat{F}(p)=\left(p^{2}-1\right)^{2}$, i.e., $F(p)=\widehat{F}(p)$ if $|p| \geq 1$ and $F(p)=0$ for $|p|<1$. When $f \in L^{2}(0,1)$, strict convexity of the 
low-order term guarantees that $J$ is strictly convex. Indeed, for $0 \leq \lambda \leq 1$,

$$
J(\lambda u+(1-\lambda) v) \leq \lambda J(u)+(1-\lambda) J(v)-\lambda(1-\lambda)\|u-v\|_{L^{2}(0,1)}^{2} .
$$

Classical results from the calculus of variations then show that $J$ has a unique minimizer. The following theorem characterizes the degenerate set where $F\left(u^{\prime}\right)$ vanishes and establishes regularity of the solution on this set.

Theorem 2.1 (Regularity). Let $f \in H^{1}(0,1)$, and assume there is an interval $[a, b] \subset[0,1](a \leq b)$ such that

$$
[a, b]=\left\{x \in[0,1] \mid-1 \leq f^{\prime}(x) \leq 1\right\} .
$$

Then there is a subinterval $[\alpha, \beta] \subset[a, b]$ such that $[\alpha, \beta] \subset E$ and $|E \backslash[\alpha, \beta]|$ $=0$, where

$$
E=\left\{x \in(0,1) \mid-1 \leq u^{\prime}(x) \leq 1\right\} .
$$

Moreover, $u(x)=f(x)$ on $[\alpha, \beta]$.

Proof. The Euler-Lagrange equation for the variational problem (1) is

$$
-\frac{d}{d x} F^{\prime}\left(u^{\prime}\right)+2(u-f)=0 \text { in } W^{-1,4 / 3}(0,1),
$$

implying $F^{\prime}\left(u^{\prime}\right) \in H^{1}(0,1)$. Clearly, $F^{\prime}\left(u^{\prime}(x)\right)=0$ for $x \in E$, so that $(d / d x) F^{\prime}\left(u^{\prime}(x)\right)=0$ for a.e. $x \in E$. The Euler-Lagrange equation then shows $u(x)=f(x)$, and hence $u^{\prime}(x)=f^{\prime}(x)$ for a.e. $x \in E$. Since $\left|f^{\prime}(x)\right|>1$ on $(0,1) \backslash[a, b]$, it follows that $|E \backslash[a, b]|=0$. Define

$$
\alpha=\inf \{x \in[a, b] \mid f(x)=u(x)\} \quad \text { and } \quad \beta=\sup \{x \in[a, b] \mid f(x)=u(x)\} .
$$

Certainly $|E \backslash[\alpha, \beta]|=0$, so it suffices to show that $u(x)=f(x)$ for a.e. $x \in[\alpha, \beta]$. This is easily deduced from the functional $J$ itself. If $u(x)$ does not equal $f(x)$ on $[\alpha, \beta]$, one could lower the energy by making it so.

2.2. Discrete problem. We approximate the variational problem using a piecewise linear approximation to $u$ on $(0,1)$, and, for ease of exposition, assume that the mesh points are equally spaced a distance $h=1 / N$ apart. Denote the associated space of piecewise linear functions that vanish at $x=0$ and $x=1$ by $U_{h}$, and for any continuous function $f$ on $[0,1]$ denote by $\bar{f}_{h}$ the piecewise constant function that takes on the values $f_{i}=f(i h)$ at the nodes $x_{i}=i h, i=0, \ldots, N$. We then approximate $J$ by

$$
\begin{aligned}
J_{h}\left(u_{h}\right) & =\int_{0}^{1} F\left(u_{h}^{\prime}\right)+\left(\bar{u}_{h}-\bar{f}_{h}\right)^{2} \\
& =\sum_{i=1}^{N} h F\left(\frac{u_{i}-u_{i-1}}{h}\right)+\sum_{i=1}^{N-1} h\left(u_{i}-f_{i}\right)^{2}+\frac{h}{2}\left(f_{0}^{2}+f_{N}^{2}\right)
\end{aligned}
$$

$\left(u_{i}=u_{h}(i h)\right.$ and $\left.f_{i}=f(i h)\right)$ and minimize $J_{h}$ over $U_{h}$. Analogous to the continuous problem, $J_{h}$ is strictly convex in the discrete $L^{2}$ norm, so that minima of these discrete variational problems exist and are unique. We begin by establishing that the sequence $\left\{u_{h}\right\}_{h>0}$ is a minimizing sequence for the original problem. 
Lemma 2.2. (i) If $f \in C(0,1)$, then $J\left(u_{h}\right) \rightarrow J(u)$.

(ii) If $f \in H^{2}(0,1)$, then

$$
J\left(u_{h}\right)-J(u) \leq C h^{2}+\inf _{v_{h} \in U_{h}}\left\|u-v_{h}\right\|_{W^{1,4}(0,1)}^{2}
$$

Proof. Let $v_{h}$ be the best projection of $u$ onto $U_{h} \subset U$ [2]. Then

$$
\begin{aligned}
0 \leq & J\left(u_{h}\right)-J(u)=J\left(u_{h}\right)-J_{h}\left(u_{h}\right)+J_{h}\left(u_{h}\right)-J(u) \\
\leq & J\left(u_{h}\right)-J_{h}\left(u_{h}\right)+J_{h}\left(v_{h}\right)-J(u) \\
\leq & \int_{0}^{1}\left(u_{h}-f\right)^{2}-\left(\bar{u}_{h}-\bar{f}_{h}\right)^{2}+\left(\bar{v}_{h}-\bar{f}_{h}\right)^{2}-\left(v_{h}-f\right)^{2} \\
& +\int_{0}^{1}\left\{F^{\prime}\left(u^{\prime}\right)\left(v_{h}^{\prime}-u^{\prime}\right)+2(u-f)\left(v_{h}-u\right)\right\} \\
& +\int_{0}^{1} F^{\prime \prime}(\xi)\left(v_{h}^{\prime}-u^{\prime}\right)^{2}+\left(v_{h}-u\right)^{2} \\
\leq & \int_{0}^{1}\left(u_{h}-f\right)^{2}-\left(\bar{u}_{h}-\bar{f}_{h}\right)^{2}+\left(\bar{v}_{h}-\bar{f}_{h}\right)^{2}-\left(v_{h}-f\right)^{2} \\
& +\int_{0}^{1} F^{\prime \prime}(\xi)\left(v_{h}^{\prime}-u^{\prime}\right)^{2}+\left(v_{h}-u\right)^{2},
\end{aligned}
$$

where $\xi(x) \in\left(u^{\prime}(x), v_{h}^{\prime}(x)\right)$ by Taylor's theorem, and the Euler-Lagrange equation was used to derive the last line. The theorem now follows from the fact that integration of the piecewise constant functions $\bar{f}$ etc. is equivalent to the trapezoid quadrature rule, which converges for any continuous function and has quadrature errors of size $C h^{2}$ for both piecewise linear functions bounded in $H^{1}(0,1)$, and functions in $H^{2}(0,1)$.

Lemma 2.3. Let $u_{h}$ be the solution of the discrete problem and $f \in H^{2}(0,1)$; then

$$
\left\|u-u_{h}\right\|_{L^{2}(0,1)}^{2} \leq J\left(u_{h}\right)-J(u)
$$

Proof. Direct computation shows

$$
\begin{aligned}
J\left(u_{h}\right)-J(u)= & \int_{0}^{1}\left(u_{h}-u\right)^{2}+F^{\prime \prime}(\xi)\left(u_{h}^{\prime}-u^{\prime}\right)^{2} \\
& +\int_{0}^{1}\left\{F^{\prime}\left(u^{\prime}\right)\left(u_{h}^{\prime}-u^{\prime}\right)+2(u-f)\left(u_{h}-u\right)\right\} .
\end{aligned}
$$

Note that $F^{\prime \prime} \geq 0$ since $F$ is convex, and that the last line vanishes by the Euler-Lagrange equation.

2.3. Convergence of the discrete scheme. While strict convexity of $J$ in the $L^{2}(0,1)$ norm implies that a minimizing sequence will converge strongly in this norm, a less trivial observation is that the derivatives will converge on the set where $F(u)$ is strictly convex, i.e., on $(0,1) \backslash E$. We show this using recent results of Kinderlehrer and Pedregal [8] who establish the existence of Young measures for minimizing sequences bounded in $W^{1, p}$ for finite $p$. The following theorem is a special case of their result and is true in much greater generality than stated here. 
Theorem 2.4 (Kinderlehrer and Pedregal). Let $\left\{v_{k}\right\}$ be a weakly convergent sequence in $W^{1,4}(0,1)$ with limit $v$ satisfying

$$
\int_{0}^{1} F\left(v_{k}^{\prime}\right) \rightarrow \int_{0}^{1} F\left(v^{\prime}\right)
$$

Then, for a suitable subsequence, there is a corresponding Young measure $\nu=$ $\left\{\nu_{x}\right\}_{x \in(0,1)}$ such that $\psi\left(v_{k}^{\prime}\right) \rightarrow \bar{\psi}$ in $L^{1}(0,1)$ for any continuous function $\psi$ satisfying $|\psi(s)| \leq C\left(1+|s|^{4}\right)$, where

$$
\bar{\psi}(x)=\left\langle\psi, \nu_{x}\right\rangle \equiv \int_{\mathbb{R}} \psi(s) d \nu_{x}(s) .
$$

Lemma 2.5. Let $\left\{u_{h}\right\}_{h>0}$ denote the solutions of the discrete problems (2); then

$$
\int_{(0,1) \backslash E}\left|u_{h}^{\prime}-u^{\prime}\right|^{4} \rightarrow 0 .
$$

Proof. We apply the above theorem to the minimizing sequence $\left\{u_{h}\right\}$ obtained in $\S 2.2$. Since $J$ is lower semicontinuous, we obtain

$$
\begin{aligned}
J(u) & =\lim _{h \rightarrow 0} J\left(u_{h}\right), \\
\int_{0}^{1} F\left(u^{\prime}\right)+(u-f)^{2} & =\lim _{h \rightarrow 0} \int_{0}^{1} F\left(u_{h}^{\prime}\right)+\left(u_{h}-f\right)^{2}, \\
\int_{0}^{1} F\left(u^{\prime}\right) & =\lim _{h \rightarrow 0} \int_{0}^{1} F\left(u_{h}^{\prime}\right) .
\end{aligned}
$$

Putting $\psi=F$, we conclude that $F\left(u^{\prime}\right)=\bar{F}$. Next, Jensen's inequality gives

$$
F\left(u^{\prime}(x)\right)=F\left(\int_{\mathbb{R}} s d \nu_{x}(s)\right) \leq \int_{\mathbb{R}} F(s) d \nu_{x}(s)=\bar{F}(x),
$$

and additionally, if $F$ is strictly convex at $u^{\prime}(x)$ (i.e., $F^{\prime \prime}>0$ ), then $F\left(u^{\prime}(x)\right.$ ) $<\bar{F}(x)$ unless $\nu_{x}=\delta_{u^{\prime}(x)}$. Since $F\left(u^{\prime}\right)=\bar{F}$, it follows that $\nu_{x}=\delta_{u^{\prime}(x)}$ at points where $\left|u^{\prime}(x)\right|>1$, i.e., on $(0,1) \backslash E$.

We now choose $\psi(s)=|s|^{4}$ to obtain

$$
\int_{(0,1) \backslash E}\left|u_{h}^{\prime}(x)\right|^{4} d x \rightarrow \int_{(0,1) \backslash E}\left\langle|\cdot|^{4}, \nu_{x}\right\rangle d x=\int_{(0,1) \backslash E}\left|u^{\prime}(x)\right|^{4} d x .
$$

Since $L^{4}((0,1) \backslash E)$ is a uniformly convex space and $u_{h}^{\prime}$ converges weakly to $u^{\prime}$, the convergence of the norms implies strong convergence.

Theorem 2.6. Suppose the inhomogeneous term, $f$, in (1) is continuously differentiable and there is a pair of real numbers $a \leq b$ such that

$(a, b)=\left\{x \in(0,1) \mid-1<f^{\prime}(x)<1\right\}, \quad\left\{x \in[0,1]|| f^{\prime}(x) \mid=1\right\} \subset\{a, b\}$.

Let $\left\{u_{h}\right\}_{h>0}$ denote the solutions of the discrete problems (2); then

$$
\int_{0}^{1}\left|u_{h}^{\prime}-u^{\prime}\right|^{4} \rightarrow 0
$$

Proof. Observe that it is sufficient to show that the derivatives converge on the set $[\alpha, \beta]$ given by Theorem 2.1. The proof proceeds in three steps. In the first step we show that if $F^{\prime}\left(u_{h}^{\prime}\right)=0$ on two elements $\left(x_{i-1}, x_{i}\right)$ and $\left(x_{k-1}, x_{k}\right)$ 
in $[\alpha, \beta]$, then $f_{j}=u_{j}$ for all $i \leq j \leq k-1$. We next show that for arbitrary $\varepsilon>0, F^{\prime}\left(u_{h}\right)$ must be zero on at least one element in each of the intervals $[\alpha, \alpha+\varepsilon]$ and $[\beta-\varepsilon, \beta]$, for $h$ sufficiently small. Finally, we use Theorem 2.4 to show that errors on intervals of size $\varepsilon$ are negligible. (2),

We begin by considering the Euler-Lagrange equation for the discrete problem

$$
F^{\prime}\left(u_{i}^{\prime}\right)-F^{\prime}\left(u_{i+1}^{\prime}\right)+2 h\left(u_{i}-f_{i}\right)=0, \quad 1 \leq i \leq N-1,
$$

where $u_{i}^{\prime}=\left(u_{i}-u_{i-1}\right) / h$ is the derivative on the $i$ th interval. Summing these equations from $i$ to $j-1$ gives

$$
F^{\prime}\left(u_{i}^{\prime}\right)-F^{\prime}\left(u_{j}^{\prime}\right)+2 h \sum_{l=i}^{j-1}\left(u_{l}-f_{l}\right)=0 .
$$

Now suppose that $F^{\prime}\left(u_{i}^{\prime}\right)=F^{\prime}\left(u_{k}^{\prime}\right)=0$ for elements $i$ and $k$ contained in $[\alpha, \beta]$. We show that $u_{j}=f_{j}$ for $i \leq j \leq k-1$. Suppose not. Then, without loss of generality, assume that $F^{\prime}\left(u_{i+1}^{\prime}\right) \neq 0$ and consider the situation where $F^{\prime}\left(u_{i+1}^{\prime}\right)>0$. Then $u_{i+1}^{\prime}>1$, and the Euler-Lagrange equation gives

$$
u_{i}-f_{i}=F^{\prime}\left(u_{i+1}^{\prime}\right) / 2 h>0 \text {. }
$$

Let $j$ be the first index in $\{i+1, i+2, \ldots, k\}$ where $F^{\prime}\left(u_{j}^{\prime}\right) \leq 0$. For each $i+1 \leq l<j$, we have $u_{l}^{\prime}>1$, so that $u_{l+1}-f_{l+1}>u_{l}-f_{l}$ (recall $\left.\left|f^{\prime}\right| \leq 1\right)$, i.e.,

$$
u_{j}-f_{j}>u_{j-1}-f_{j-1}>\cdots>u_{i}-f_{i}>0 .
$$

Putting this into the summed Euler-Lagrange equation gives a sum of strictly positive quantities being zero, a contradiction. A similar argument for $F^{\prime}\left(u_{i+1}^{\prime}\right)$ $<0$ excludes this possibility too.

We next show that, for $h$ sufficiently small, there are always elements near $\alpha$ and $\beta$ where $F^{\prime}$ vanishes.

Consider an arbitrary interval $I=[x, x+\varepsilon] \subset(\alpha, \beta)$. Suppose that $F^{\prime}\left(u_{h}^{\prime}\right)$ does not vanish in $I$. We then claim that it must change sign. If it did not, suppose that it was positive (or negative), so that $u_{h}^{\prime}>1$ (or $<-1$ ). Since, for some $\delta>0,\left|f^{\prime}\right| \leq 1-\delta$ on $[x, x+\varepsilon]$, it follows that $u_{h}-f$ is monotone and

$$
\int_{x}^{x+\varepsilon}\left(u_{h}-f\right)^{2} \geq \varepsilon^{3} \delta^{2} / 12=c(\varepsilon) .
$$

Since $u=f$ on $[\alpha, \beta]$, it follows that $\left\|u_{h}-u\right\|_{L^{2}(0,1)}^{2} \geq c(\varepsilon)$, which cannot persist for small $h$ since $u_{h} \rightarrow u$ in $L^{2}(0,1)$.

We now show that the oscillations implied by the above argument are incompatible with the discrete Euler-Lagrange equation. Again assume that $F^{\prime}\left(u_{h}^{\prime}\right)$ does not vanish on $[x, x+\varepsilon] \subset(\alpha, \beta)$, and assume that $h$ is sufficiently small to guarantee $F^{\prime}\left(u^{\prime}\right)$ changes sign twice in this interval (repeating the above argument with $\varepsilon / 2$ if necessary). Note that at nodes where $F^{\prime}\left(u^{\prime}\right)$ changes from positive to negative, the Euler-Lagrange equation gives $u_{i}<f_{i}$, and where it changes from negative to positive, $u_{i}>f_{i}$. This immediately leads to a contradiction if we consider what happens between two consecutive sign changes in $F^{\prime}\left(u^{\prime}\right)$, say at nodes $x_{i}$ and $x_{j}$. Suppose for the sake of argument that $F^{\prime}\left(u^{\prime}\right)$ is positive for $x \leq x_{i}$, negative for $x \in\left(x_{i}, x_{j}\right)$, and positive for $x \geq x_{j}$. 
Between $x_{i}$ and $x_{j}, u_{h}^{\prime}<-1$ while $\left|f^{\prime}\right| \leq 1$, implying $u_{h}-f$ is monotone decreasing. However, the Euler-Lagrange equation implies $u_{i}-f_{i}<0$ and $u_{j}-f_{j}>0$, a contradiction.

The above two paragraphs establish that if $\varepsilon>0$ and $[x, x+\varepsilon] \subset(\alpha, \beta)$, then $F^{\prime}\left(u^{\prime}\right)=0$ on at least one element in $[x, x+\varepsilon]$ for $h$ sufficiently small. Applying this to intervals of the form $[\alpha+\varepsilon, \alpha+2 \varepsilon]$ and $[\beta-2 \varepsilon, \beta-\varepsilon]$ shows that, for $h$ sufficiently small, $u_{i}=f_{i}=u\left(x_{i}\right)$ for all nodes in $[\alpha+2 \varepsilon, \beta-2 \varepsilon]$, and hence $u_{h}^{\prime} \rightarrow u^{\prime}$ on all such intervals.

Finally, we establish that, for any $x \in(0,1), \int_{x}^{x+\varepsilon}\left|u_{h}^{\prime}\right|^{4} \rightarrow 0$ uniformly in $h$ as $\varepsilon \rightarrow 0$. This follows directly from the fact that $\left|u_{h}^{\prime}\right|^{4}$ converges weakly in $L^{1}(0,1)$, a consequence of Theorem 2.4 .

\section{RATES OF CONVERGENCE}

While the proof above provides insight into the behavior of the numerical scheme on the degenerate region, very little is known about the behavior on the complement, where the problem is "almost" degenerate, i.e., when $\left|u^{\prime}\right| \simeq 1$. To accommodate this, we introduce the following weighted norm on $W_{0}^{1,4}(0,1)$ :

$$
\|v\|^{2} \equiv \int_{(0,1) \backslash E}\left(u^{\prime 2}-1\right) v^{\prime 2}+\int_{E} v^{\prime 2}+\int_{0}^{1} v^{2}
$$

(recall that $\left.E=\left\{x:\left|u^{\prime}(x)\right| \leq 1\right\}\right)$. Let $M$ denote the completion of $W_{0}^{1,4}(0,1)$ under this norm, and extend the definition of $J$ to all of $M$ by

$$
J(v)= \begin{cases}\int_{0}^{1} F\left(v^{\prime}\right)+(v-f)^{2} & \text { if } v \in W_{0}^{1,4}(0,1), \\ \infty & \text { otherwise }\end{cases}
$$

This extension by infinity guarantees that $J: M \rightarrow \mathbb{R} \cup\{\infty\}$ is lower semicontinuous, so that the following lemma, due to Ekeland, is applicable.

Lemma 3.1 (Ekeland). Let $M$ be a complete metric space with metric $d$, and let $J: M \rightarrow \mathbb{R} \cup\{\infty\}$ be lower semicontinuous, bounded from below, and not identically infinite. Then for any $\varepsilon, \delta>0$ and any $u \in M$ with

$$
J(u) \leq \inf _{M} J+\varepsilon^{2},
$$

there is an element $v \in M$ strictly minimizing the functional

$$
J_{v}(w)=J(w)+\frac{\varepsilon^{2}}{\delta} d(v, w) .
$$

Moreover,

$$
J(v) \leq J(u), \quad d(u, v) \leq \delta .
$$

A proof of this lemma may be found in [4]. With $u_{h}$ denoting the solution of (2), Lemma 2.3 gives

$$
J\left(u_{h}\right) \leq J(u)+c(h)^{2}=\inf _{M} J+c(h)^{2},
$$

where $c(h) \leq C h$ if the minimizer $u \in W^{2,4}(0,1)$. Application of Ekeland's lemma with $\varepsilon=\delta=c(h)$ gives $v_{h} \in M$ such that $\left\|u_{h}-v_{h}\right\| \leq c(h)$ and 
$J\left(v_{h}\right) \leq J\left(u_{h}\right)<\infty$, implying $v_{h} \in W_{0}^{1,4}(0,1)$. Also, since $v_{h}$ is a minimizer of the modified problem, we obtain

$$
J\left(v_{h}\right) \leq J\left(v_{h}+w\right)+c(h)\|w\|, \quad w \in M,
$$

or, replacing $w$ by $\varepsilon w$ for $w \in W_{0}^{1,4}(0,1)$ and letting $\varepsilon \rightarrow 0$ gives an EulerLagrange inequality

$$
\int_{0}^{1} F^{\prime}\left(v_{h}^{\prime}\right) w^{\prime}+2\left(v_{h}-f\right) w \leq c(h)\|w\|, \quad w \in W_{0}^{1,4}(0,1) .
$$

Subtracting this from the Euler-Lagrange equation for the original problem gives

$$
\int_{0}^{1}\left[F^{\prime}\left(u^{\prime}\right)-F^{\prime}\left(v_{h}^{\prime}\right)\right] w^{\prime}+2\left(u-v_{h}\right) w \leq c(h)\|w\|, \quad w \in W_{0}^{1,4}(0,1) .
$$

In particular, putting $w=u-v_{h}$ gives

$$
\int_{0}^{1}\left[F^{\prime}\left(u^{\prime}\right)-F^{\prime}\left(v_{h}^{\prime}\right)\right]\left(u^{\prime}-v_{h}^{\prime}\right)+2\left(u-v_{h}\right)^{2} \leq c(h)\left\|u-v_{h}\right\| .
$$

This estimate will yield a rate of convergence of $v_{h}$ and hence of $u_{h}$ in the given norm $\|\cdot\|$. The following two inequalities pertaining to $F$ will be required in the theorem below. If $|b|>1$ and $|a| \geq 1$, then

$$
\begin{aligned}
{\left[F^{\prime}(b)-F^{\prime}(a)\right](b-a) } & =2\left[(b+a)^{2}+\left(b^{2}+a^{2}-2\right)\right](b-a)^{2} \\
& \geq 2\left(b^{2}-1\right)(b-a)^{2},
\end{aligned}
$$

and if $|b|>1,|a| \leq 1$,

$$
\begin{aligned}
{\left[F^{\prime}(b)-F^{\prime}(a)\right](b-a) } & =4\left(b^{2}-1\right) b(b-a) \\
& =2\left(b^{2}-1\right)\left[(b-a)^{2}+b^{2}-a^{2}\right] \\
& \geq 2\left(b^{2}-1\right)(b-a)^{2} .
\end{aligned}
$$

Theorem 3.2. Let $\|\cdot\|$ be the norm defined above, and let $E \simeq[\alpha, \beta]$ denote the degenerate set as in Theorem 2.1. If the inhomogeneous term $f \in H^{2}(0,1)$ satisfies $f^{\prime}(x) \leq 1-\delta$ in neighborhoods of $\alpha$ and $\beta$, then

$$
\left\|u-u_{h}\right\| \leq C c(h)^{1 / 3} \text {. }
$$

Otherwise, if $\left|f^{\prime}(\alpha)\right|=1$ and/or $\left|f^{\prime}(\beta)\right|=1$, and $0<c \leq f^{\prime \prime}$ or $f^{\prime \prime} \leq-c<0$ in a neighborhood of $\alpha$ and/or $\beta$, then

$$
\left\|u-u_{h}\right\| \leq C c(h)^{1 / 5},
$$

where $c(h)^{2}=J\left(u_{h}\right)-J(u) \leq C h^{2}+\inf _{w_{h} \in U_{h}}\left\|w_{h}-u\right\|_{U}^{2}$.

Proof. We begin by first estimating the error on the nondegenerate set $(0,1) \backslash E$ where $\left|u^{\prime}\right|>1$. The inequalities satisfied by $F$ show that

$$
\int_{[0,1] \backslash E}\left[F^{\prime}\left(u^{\prime}\right)-F^{\prime}\left(v_{h}^{\prime}\right)\right]\left(u^{\prime}-v_{h}^{\prime}\right) \geq 2 \int_{[0,1] \backslash E}\left(u^{\prime 2}-1\right)\left(u^{\prime}-v_{h}^{\prime}\right)^{2} .
$$

We next estimate the error on the degenerate set $E=[\alpha, \beta]$ where $\left|u^{\prime}\right| \leq 1$. The proof of Theorem 2.6 showed that there exists $\varepsilon(h)>0$ such that the discrete nodal values of $u_{h}$ exactly interpolated $u$ on $E_{\varepsilon}=[\alpha+2 \varepsilon(h), \beta-$ $2 \varepsilon(h)]$, and in this instance standard approximation theory shows that

$$
\left\|u^{\prime}-u_{h}^{\prime}\right\|_{L^{2}\left(E_{\varepsilon}\right)}=\left\|f^{\prime}-u_{h}^{\prime}\right\|_{L^{2}\left(E_{\ell}\right)} \leq\left\|f^{\prime \prime}\right\|_{L^{2}\left(E_{\ell}\right)} h \leq C h .
$$


The triangle inequality and Lemma 3.1 then give

$$
\left\|u^{\prime}-v_{h}^{\prime}\right\|_{L^{2}\left(E_{\varepsilon}\right)} \leq\left\|u^{\prime}-u_{h}^{\prime}\right\|_{L^{2}\left(E_{\varepsilon}\right)}+\left\|u_{h}^{\prime}-v_{h}^{\prime}\right\|_{L^{2}(E)} \leq C h+c(h) .
$$

The error on the intervals of size $2 \varepsilon$ is estimated by dividing them into two pieces, $E^{+}$where $\left|v_{h}^{\prime}\right|>\sqrt{2}$ and $E^{-}$where $\left|v_{h}^{\prime}\right| \leq \sqrt{2}$. Since $\left|u^{\prime}\right| \leq 1$, it follows that

$$
\left\|u^{\prime}-v_{h}^{\prime}\right\|_{L^{2}\left(E^{-}\right)}^{2} \leq C \varepsilon(h)
$$

and

$$
\int_{E^{+}}\left[F^{\prime}\left(u^{\prime}\right)-F^{\prime}\left(v_{h}^{\prime}\right)\right]\left(u^{\prime}-v_{h}^{\prime}\right)=\int_{E^{+}}\left(v_{h}^{\prime 2}-1\right) v_{h}^{\prime}\left(v_{h}^{\prime}-u^{\prime}\right) \geq\left\|u^{\prime}-v_{h}^{\prime}\right\|_{L^{2}\left(E^{+}\right)}^{2} .
$$

Combining the above estimates with equation (4) gives

$$
\begin{aligned}
\left\|u-v_{h}\right\|^{2} \leq & (1 / 2) \int_{0}^{1}\left\{\left[F^{\prime}\left(u^{\prime}\right)-F^{\prime}\left(v_{h}^{\prime}\right)\right]\left(u^{\prime}-v_{h}^{\prime}\right)+2\left(u-v_{h}\right)^{2}\right\} \\
& +\left\|u^{\prime}-v_{h}^{\prime}\right\|_{L^{2}\left(E_{\varepsilon}\right)}^{2}+\left\|u^{\prime}-v_{h}^{\prime}\right\|_{L^{2}\left(E^{-}\right)}^{2} \\
\leq & (1 / 2) c(h)\left\|u-v_{h}\right\|+[C h+c(h)]^{2}+C \varepsilon(h) \\
\leq & C\left[c(h)^{2}+h^{2}+\varepsilon(h)\right] .
\end{aligned}
$$

Finally we estimate $\varepsilon(h)$. Equation (3) gives

$$
\varepsilon^{3} \delta^{2} \leq C\left\|u-u_{h}\right\|_{L^{2}(0,1)}^{2} \leq C c(h)^{2} .
$$

If $\left|f^{\prime}\right|<1-\delta$ on neighborhoods of $\alpha$ and $\beta$, this gives an estimate

$$
\varepsilon \leq C c(h)^{2 / 3}
$$

otherwise, if $\left|f^{\prime}(\alpha)\right|=1$ or $\left|f^{\prime}(\beta)\right|=1$, the proof of Theorem 1 shows that it is necessary to estimate $f^{\prime}$ on intervals of the form $[\alpha+\varepsilon, \alpha+2 \varepsilon]$ and $[\beta-2 \varepsilon, \beta-\varepsilon]$. The assumption on the second derivative of $f$ will then show that $\left|f^{\prime}\right| \leq 1-c \varepsilon$ on these intervals, giving

$$
\varepsilon \leq C c(h)^{2 / 5} \text {. }
$$

\section{Application to calculation of Young measures}

Recently, the authors have proposed an algorithm to calculate generalized solutions of nonconvex variational problems. A generalized solution is a pair $(u, \nu)$, where $u$ is the weak limit of a minimizing sequence and $\nu$ is the associated Young measure. In general, $u$ will be a slowly varying function, and $\nu$ characterizes the fine oscillations that develop in the minimizing sequence. This motivates the development of algorithms to calculate these quantities, since they can be well represented on finite meshes in contrast with the minimizing sequences, which develop infinitely many oscillations. Consider the inhomogeneous Young problem given by

$$
\widehat{J}(u)=\int_{0}^{1} \widehat{F}\left(u^{\prime}\right)+(u-f)^{2}, \quad u \in U=W^{1,4}(0,1),
$$


where $\widehat{F}$ is the double-well potential shown in Figure 1a. If $(u, \nu)$ is the generalized solution, then $u$ solves the convexified problem (1) and ${ }^{1}$

$$
\nu_{x}= \begin{cases}\delta_{u^{\prime}(x)} & \text { if }\left|u^{\prime}(x)\right| \geq 1 \\ \lambda(x) \delta_{+1}+(1-\lambda(x)) \delta_{-1} & \text { if }\left|u^{\prime}(x)\right|<1\end{cases}
$$

where $\lambda(x)=\left(1+u^{\prime}(x)\right) / 2$. Collins, Kinderlehrer, and Luskin [3] consider the numerical approximations obtained by directly minimizing $\widehat{J}$ and show that infinitely many oscillations will develop as the mesh is refined; moreover, if the oscillations are suitably averaged, it is possible to estimate $\lambda$.

For this particular problem, the algorithm proposed by the authors in [9] reduces to approximating minima of

$$
I(u, \lambda, b)=\int_{0}^{1} \lambda \widehat{F}\left[u^{\prime}-(1-\lambda) b\right]+(1-\lambda) \widehat{F}\left[u^{\prime}+\lambda b\right]+(u-f)^{2},
$$

where $u \in W^{1,4}(0,1), b \in L^{4}(0,1)$, and $\lambda \in L^{\infty}(0,1)$ is subject to the constraint $0 \leq \lambda \leq 1$. The corresponding discrete problem is to minimize

$$
I\left(u_{h}, \lambda_{h}, b_{h}\right)=\int_{0}^{1} \lambda_{h} \widehat{F}\left[u_{h}^{\prime}-\left(1-\lambda_{h}\right) b_{h}\right]+\left(1-\lambda_{h}\right) \widehat{F}\left[u_{h}^{\prime}+\lambda_{h} b_{h}\right]+\left(\bar{u}_{h}-\bar{f}_{h}\right)^{2},
$$

over the space of piecewise linear functions for $u_{h}$ and piecewise constant functions for $\lambda_{h}$ and $b_{h}, 0 \leq \lambda_{h} \leq 1$. It is elementary to show, by first minimizing over $\lambda$ and $b$ (resp. $\lambda_{h}$ and $b_{h}$ ), that $u$ (resp. $u_{h}$ ) must solve the convexified problem (1) (resp. (2)). It then follows that the strong convergence and rates given by Theorems 2.6 and 3.2 for the convexified problem are enjoyed by $\left\{u_{h}\right\}_{h>0}$, the solutions of the approximate generalized problem.

The Young measure implied by this algorithm is

$$
\nu=\lambda \delta_{u^{\prime}-(1-\lambda) b}+(1-\lambda) \delta_{u^{\prime}+\lambda b}
$$

Note that the representation of this measure is not unique. Indeed, we can change the sign of $b$ if we interchange $\lambda$ with $1-\lambda$, and with $b=0, \lambda$ arbitrary, or $\lambda=0$ or 1 and $b$ arbitrary, the measure reduces to $\delta_{u^{\prime}}$. These ambiguities are eliminated if we agree to select $b \geq 0$ and $0<\lambda<1$, and consider $\lambda$ undefined when $b=0$. In this situation, $b(x), b_{h}(x) \in\{0,2\}$, so that

$$
b=2 \chi_{\left|u^{\prime}\right|<1}, \quad b_{h}=2 \chi_{\left|u_{h}^{\prime}\right|<1},
$$

and

$$
\lambda=\frac{1}{2}\left(1+u^{\prime}\right) \chi_{\left|u^{\prime}\right|<1}, \quad \lambda_{h}=\frac{1}{2}\left(1+u_{h}^{\prime}\right) \chi_{\left|u_{h}^{\prime}\right|<1},
$$

where $\chi_{(\cdot)}$ is the characteristic function of the indicated set. Since $u_{h}$ converges strongly in $W^{1,4}(0,1)$ to $u$, it follows that $\lambda_{h}$ and $b_{h}$ converge almost everywhere to $\lambda$ and $b$ (for a suitable subsequence). Since they are also bounded in $L^{\infty}(0,1)$, it follows that they converge strongly in $L^{p}(0,1), 1 \leq p<\infty$, and weakly star in $L^{\infty}(0,1)$. Convergence of $\lambda_{h}$ and $b_{h}$ then implies $\left\{\nu_{h}\right\}$ converges weakly star to $\nu$.

\footnotetext{
${ }^{1} \delta(\bullet)$ is the Dirac measure concentrated at the indicated point.
} 


\section{BIBLIOGRAPHY}

1. M. Chipot and D. Kinderlehrer, Equilibrium configurations of crystals, Arch. Rational Mech. Anal. 103 (1988), 237-277.

2. P. G. Ciarlet, The finite element method for elliptic problems, North-Holland, Amsterdam, 1978.

3. C. Collins, D. Kinderlehrer, and M. Luskin, Numerical approximation of the solution of a variational problem with a double well potential, SIAM J. Numer. Anal. 28 (1991), 321-332.

4. I. Ekeland, Convexity methods in Hamiltonian mechanics, Springer-Verlag, Berlin, 1980.

5. D. French, On the convergence of finite element approximations of a relaxed variational problem, SIAM J. Numer. Anal. 27 (1990), 419-436.

6. R. D. James, Finite deformation by mechanical twinning, Arch. Rational Mech. Anal. 77 (1981), 143-176.

7. R. D. James and D. Kinderlehrer, Frustration in ferromagnetic materials, Contin. Mech. Thermodyn. 2 (1990), 215-239.

8. D. Kinderlehrer and P. Pedregal, Weak convergence of integrands and the Young measure representation, SIAM J. Math. Anal. 23 (1992), 1-19.

9. R. A. Nicolaides and N. J. Walkington, Computation of microstructure utilizing Young measure representations, Recent Advances in Adaptive and Sensory Materials and their Applications (C. A. Rogers and R. A. Rogers, eds.), Technomic Publishing Co., Lancaster, 1992, pp. 131-141.

Department of Mathematics, Carnegie Mellon University, Pittsburgh, Pennsylvania 15213 